\title{
Efficiency application of running exercises with skandinavian sticks in the complex strength training of young biathletes
}

\author{
Talgat Sagiev ${ }^{1 *}$, Ildus Gibadullin ${ }^{2}$ \\ ${ }^{1}$ Omsk State Technical University, 644050, Omsk, Russia \\ ${ }^{2}$ Izhevsk State Technical University named after M. T. Kalashnikov, 426069, Izhevsk, Russia
}

\begin{abstract}
The authors of the article based on the systematization of data from scientific and methodological literature and analysis of training young biathletes practice developed a block-modular technology of complex strength training in the snow-free period, and also modified scandinavsky walking as a type of active activity to running exercises with Scandinavian sticks in order to develop strength endurance of the shoulder girdle muscles. Based on the conducted pedagogical experiment, the authors came to the conclusion that it is advisable to reduce cross-country training by $30-40 \%$ in the snow-free period due to the use of running exercises with Scandinavian sticks. The combination of block-modular complex strength training with Scandinavian sticks exercises allows us to most effectively increase the indicators of the shoulder girdle muscles power endurance and functional readiness of biathletes aged 13-16 years.
\end{abstract}

\section{Introduction}

In the practice of training young biathletes, many coaches in the summer are oriented towards increasing the volume of cyclic load, fill the training process with cross-country and ski-roller without monitoring heart rate indicators. It leads to the intensification and early emasculation of the teenagers body, exhaustion of its reserves and injury. The tasks of such training sessions are aimed at honing elements of cross-country skiing techniques, increasing functional readiness, speed and power endurance, mainly by using special exercises [1, 2, 3]. In scientific and methodological literature sources, on the contrary, there is a need for a variety of training tools and methods. Because the rich content strength exercises, borrowed from the field of fitness, gymnastics, powerlifting on a variety of muscle groups allow to avoid the uniformity and monotony of young athlete training load in cyclic sports, contribute to the harmonious development of power endurance. It beneficially affects the functional readiness $[4,7$, $8,9,10]$.

Currently, nordic walking is becoming popular among the population as a fitness direction, the benefits of this motor mode have been proven by many scientists, but its main tasks are the prevention of diseases, recovery and rehabilitation of the body after injuries. With the help of sticks, experts offer a wide range of general development exercises. But athletes can also use running with shortened sticks for the development of power capabilities. The advantage of such a run is not only the similarity of the shoulder girdle muscular work with competitive running, but also the possibility and ease of regulating the load by changing the frequency and effort of repulsions. And also, unlike ski-roller training, a special track is not required for conducting classes, physical work is less intensive, and overcoming the ascents with the help of push-offs with sticks does not initially require a high level of strength training from the athlete. Running with shortened sticks does not have the sameт tasks as ski walking and jumping simulations with longer sticks [5,6]. However, as a new type of skiers and biathletes motor activity, it requires theoretical and experimental justification.

Goal: experimentally test the effectiveness of using block-modular complex strength training in the training process of the preparatory period in combination with Scandinavian sticks running exercises in biathletes aged 1316 years in order to develop strength endurance of the shoulder girdle muscles.

\section{Materials and Methods}

The research used the following methods: analysis and generalization of scientific and methodological literature, pedagogical observation, pedagogical testing, pedagogical experiment, methods of mathematical statistics.

The study was organized on the basis of "SSOR biathlon" Izhevsk, Russia. In the period from April to October 2019, a pedagogical experiment was organized, in which 60 biathletes aged 13-16 took part. Four groups of 20 biathletes were formed, i.e. KG and EG biathletes - athletes of 13-14 years of age, KG and EG biathletes-athletes of 15-16 years of age. For representatives of the control group (hereinafter referred to as $\mathrm{KG}$ ) in the training process

\footnotetext{
* Corresponding author: n222omsktalgat@yandex.ru
} 
implemented modular technology comprehensive strength training, the representatives of the experimental group (the EG) in addition to the implementation of modular technology integrated strength training has additionally been introduced Jogging exercises with the Nordic sticks by reducing cross-country training by 30-40\%, mainly athletics running low and medium intensity during the snowless period of the training.

Developed model modular integrated strength training, was distinguished by the organization of the training process in the form of block structure, within each unit was based accented development in the individual training the specific component (module) power endurance, applying tools, methods and orientation of training loads appropriate to the age peculiarities, the task blocks, the period of the training phase, as well as the specifics of the motor activity.

\section{Results and Discussion}

Running with shorter sticks (Scandinavian sticks) has an advantage in contrast to running with ski poles - a shorter step length increases the frequency of repulsions. It which reduces the speed of running (the intensity of the load), exerting an accentuated force load on the shoulder girdle muscles. In the course of practical implementation of this type of motor activity we modified it and defined it types of running exercises with Scandinavian sticks:

1. Exercise № 1 - with an emphasis on the development of the deltoid, thoracic and broadest back muscles. During running, the legs are repositioned mainly due to the extension of the ankle joint muscles, the torso is tilted forward and is 50-55 degrees relative to the horizontal plane. It seems that the legs are dragging behind the torso (drawing 1). The forward movement is performed in short steps. The distance between the heel of the front leg and the toe of the back leg should be about 25-35 centimeters. "Take-out" and setting the stick occurs just as in the alternate two-step classic move, but to finish the repulsion stick should be at the moment when the wrist is at the level of the pelvis (or not bringing the wrist to the pelvis $5-10 \mathrm{~cm}$ ), thereby the hand does not have time to straighten completely in the elbow joint.

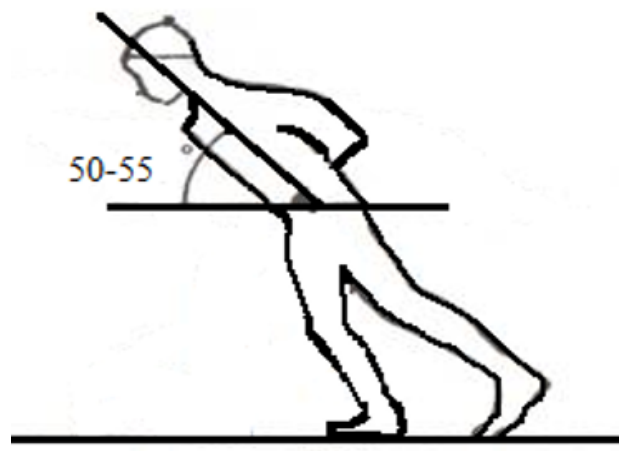

Fig. 1. Biathlete's body position when performing the exercise with scandinavian sticks №1.

2. Exercise №2 - with an emphasis on the triceps brachii and broadest back muscles. The torso is slightly tilted forward and is 75-80 degrees relative to the horizontal plane. The movement of the athlete's body forward-up is mainly due to the extension of the arm at the elbow joint. The amplitude of the hands movement is less than in exercise №1, since at the moment of placing the stick on the support, the wrist is closer to the torso. In the process of repelling the arm is fully extended at the elbow joint, in the final phase of repelling the wrist is located behind the pelvis of the athlete (at this point, the stick and the straightened arm should form a straight line). During running, the legs are slightly bent at the knees and slightly extended forward. So the athlete assumes an almost vertical position of the body. It gives resistance to the triceps shoulder muscle.

3. Exercise №3 - high-speed running with sticks aimed at developing anaerobic power endurance and movement frequency. It is performed as a normal run, the consistency of the hands and feet movements is identical to the alternate two-step classic move.

In the preparatory period we recommend using running exercises with Scandinavian sticks observing the following algorithm of training sessions methodical planning:

1) In April, after completing the transition period at the stage of "pull-in" classes, it is recommended to use scandinavian walking on flat terrain, as well as learn types of running exercises with scandinavian sticks. As an athlete develops motor skills, he should increase the frequency of cycles and switch to running, using a variable method of training.

2) In May running is applied with the stick sand on the flat ground surface with the implementation of the accelerations for short periods. Acceleration is performed with a high frequency of repulsions with the hands (conditionally - the legs do not take part in almostall, but are simply repositioned). There is no flight phase of running and the leg is almost not bent at the knee joint. 
3) In June, it is advisable to switch to variable terrain. It should include Nordic walking in the ascent, in combination with running exercises on flat terrain and descents.

4) From July until the end of the snow-free period is recommended running with sticks on the plain and in the ascent with low intensity with the implementation of the above-described running exercises №1, №2. Since September athletes perform the same thing at medium intensity.

5) From August until the end of the snow-free training period, athletes should increase the intensity of runninga with sticks over rough terrain by periodically increasing the frequency of repulsive movements during the climb. On descents and flat terrain athletes, go to step in order to restore the organism.

6) From September until the end of the snow-free training period athletes are recommended occasionally resort to high-speed running in the ascent (use exercise №3) with alternating light running with sticks on the slopes and plains.

For young biathletes, depending on the age and level of physical fitness, the length of the main work segments on sticks in each exercise is regulated and ranked. The trainer needs to monitor the correctness of performing repulsive movements, since the effectiveness of the shoulder girdle muscles power endurance development directly depends on this. In the autumn period of training, in case of achieving a high level of strength fitness, it is recommended that athletes individually use an exercise from the power gymnastics complex on the shoulder girdle muscles before performing a high-intensity running with sticks in the lift (for example, push-ups in the prone position), in order to enhance the training effect. Running with scandinavian sticks can be used not only as an independent training tool for the main part of the lesson. It is appropriate to use it as a "final part" and "hitch" in the preparatory and final parts of training and as a recovery of the body between strength exercises.

We analyzed the heart rate indicators of biathletes aged 13-16 years old obtained during training sessions in the process of running with scandinavian sticks. Pulse indicators were recorded after ascent medium steep $\left(12^{\circ}\right)$ of 100 meters and after a continuous 5-minute run on a flat area. The average values of low, medium and high intensity pulse were established (table 1).

Table 1. Heart rate indicators of biathletes aged 13-16 years after running ascent and flat terrain

\begin{tabular}{|c|c|c|c|c|c|c|}
\hline \multirow{2}{*}{ Terrain features } & \multicolumn{3}{|c|}{ Biathletes aged 13-14 years } & \multicolumn{3}{c|}{ Biathletes 15-16 years } \\
\cline { 2 - 7 } & old 1 & 2 & 3 & 1 & 2 & 3 \\
\hline Plain & $132 \pm 3,6$ & $148 \pm 4,5$ & $174 \pm 5,7$ & $125 \pm 3,2$ & $140 \pm 3,9$ & $168 \pm 5,4$ \\
\hline $\begin{array}{c}\text { Ascent medium steep of } \\
100(\mathrm{~m})\end{array}$ & $147 \pm 4,4$ & $172 \pm 5,1$ & $184 \pm 6,3$ & $141 \pm 4,0$ & $165 \pm 5,4$ & $180 \pm 5,9$ \\
\hline
\end{tabular}

Note: 1 - low-intensity running with scandinavian sticks

2 - medium-intensity running with scandinavian sticks

3 - high-intensity running with scandinavian sticks

Table 1 shows, that ranking the intensity and terrain, the pulse of young biathletes corresponds to the restorative, supportive and developmental training loads. Thus, it can be concluded that with the help of these exercises, it is possible to solve extensive educational tasks throughout the entire preparatory period.

For the purpose of experimental verification in the training process of the preparatory period in the experimental group, running exercises with scandinavian sticks were used in experimental groups along with block-modular complex power training. In the control groups, the training process differed only in the using of low and mediumintensity cross-country training instead of running with scandinavian sticks.

Table 2. Dynamics of indicators of functional readiness and power endurance of shoulder girdle muscles of young biathletes before and after the pedagogical experiment

\begin{tabular}{|l|c|c|c|c|c|}
\hline \multirow{2}{*}{ Tests } & \multirow{2}{*}{$\begin{array}{c}\text { Grou } \\
\mathrm{p}\end{array}$} & \multicolumn{2}{|c|}{ Biathletes aged 13-14 years } & \multicolumn{2}{c|}{ Biathletes 15-16 years } \\
\cline { 3 - 6 } & $\begin{array}{c}\text { start of the } \\
\text { preparatory period }\end{array}$ & $\begin{array}{c}\text { end of the } \\
\text { preparatory period }\end{array}$ & $\begin{array}{c}\text { start of the } \\
\text { preparatory period }\end{array}$ & $\begin{array}{c}\text { end of the preparatory } \\
\text { period }\end{array}$ \\
\hline $\begin{array}{l}\text { Run 3 }(\mathrm{km}) \\
\text { ski-roller DP, } \\
\text { (s) / growth } \\
\text { rate }(\%)\end{array}$ & 1 & $835 \pm 13$ & $796 \pm 14 * / 5$ & $793 \pm 13$ & $764 \pm 12 * / 4$ \\
\cline { 2 - 6 } & 2 & $827 \pm 15$ & $771 \pm 17 * / 7,3$ & $803 \pm 16$ & $752 \pm 14 * / 6,8$ \\
\hline
\end{tabular}




\begin{tabular}{|l|c|c|c|c|c|}
\hline $\begin{array}{l}\text { Run 2 (km), } \\
\text { (s) / growth } \\
\text { rate (\%) }\end{array}$ & 1 & $485 \pm 10$ & $459 \pm 8 * / 5,7$ & $463 \pm 11$ & $434 \pm 10 * / 6$ \\
\cline { 2 - 5 } & 2 & $494 \pm 8$ & $470 \pm 9 * / 5,2$ & $469 \pm 10$ & $442 \pm 9 * / 6$ \\
\hline
\end{tabular}

Note: 1 - representatives of the control group; 2 - representatives of the experimental group; * - changes in indicators with a significant significance at $\mathrm{P}<0.05$ between the indicators at the beginning of the pedagogical experiment and at the end of the pedagogical experiment; DP - classical skiing double polling technique

It is found that 13-16 years test in "running $3(\mathrm{~km})$ on ski-rollers on classical skiing using double polling technique" of the KG and the EG group athletes have an increase in performance with a reliable importance of characterizing the strength endurance of shoulder girdle muscles. However, EG representatives have a 1.5 -fold higher growth rate.

In the studied 13-16 years biathletes, the level of functional readiness has increased, this can be seen by changes with a significant significance of indicators in the test "running $2(\mathrm{~km})$ ". Increases in indicators for KG and EG biathletes aged 13-14 and 15-16 years are almost equivalent.

\section{Conclusions}

Based on the obtained results of the conducted pedagogical experiment, it can be concluded that block-modular complex strength training of young biathletes allows to significantly increase the level of young biathletes power endurance functional readiness in the preparatory period. Increasing cross-country training by $30-40 \%$ of low and medium intensity in the snow-free period due to the use of strength exercises with scandinavian sticks in combination with block-modular complex of strength training allows athletes to most effectively strengthen the necessary muscle groups of the shoulder girdle, increase their power endurance. It is important to note this planning option does not reduce the effectiveness of young biathletes cross-country training.

\section{References}

1. D.I. Ivanov, Physical culture, sports, tourism: scientific and methodological support. Materials of the all-Russian scientific and practical conference with international participation $(182-186,2019)$

2. L.N. Korchevoy, N.S. Vishkevich, E.O. Kasprisin, Physical culture and sport in modern society $(180-185,2019)$

3. L.I. Lubysheva, D.I. Ivanov, E.K. Muraleeva, Sport and sport medicine. Materials of the II all-Russian scientific and practical with international participation conference (Tchaikovsky state Institute of physical culture, Tchaikovsky, 127-130, 2019)

4. I.A. Mokrushina, A.A. Nepryakhin, Sport and sport medicine. Material of the II all-Russian scientific and practical with international participation conference (Tchaikovsky state Institute of physical culture, Tchaikovsky, 155-160, 2019)

5. A.S. Kuznetsov, Z.M. Kuznetsova, Russian Journal of Physical Education and Sport, 14(4), 5-7 (2019)

6. T.A. Sagiev, A.S. Kovaleva, L.M. Durova, Science about person: humanitarian researches, 1(31), 131-134 (2018)

7. V.V. Semennikova, Scientific notes of the P.F. Lesgaft University, 2(180), 345-348 (2020)

8. A. Bolotin, V. Bakaev, C.U., Journal of Human Sport and Exercise, 13(2), 240-244 (2018)

9. K.S. Dunaev, S.G. Seiranov, The preparation of young biathletes in the annual cycle (National Sports Academy "Vassil Levski" Sofia, Bulgaria, 20-21, 2017)

10. L.N. Korchevoy, E.A. Gonchar, O.I. Karpushkina, Theory and Practice of Physical Culture, 6, 30 (2017) 